\title{
Performance simulation of broadband multimedia wireless networks simulation based on OPNET
}

\author{
Sameer Abdul-Sattar Lafta', Aktham Hasan Ali', Marwah M. Kareem³, \\ Yasser A. Hussein ${ }^{4}$, Adnan H. Ali ${ }^{5}$ \\ ${ }^{1}$ Middle Technical University, Technical Electronics Dept., TIT Institute, Iraq \\ ${ }^{2}$ Middle Technical University, Institute of Technology, Electronic Techniques Department, Iraq \\ ${ }^{3,4}$ Faculty of Engineering \& Built Environment, Universiti Kebangsaan Malaysia, Malaysia \\ ${ }^{3}$ Computer Techniques Engineering, Al-Israa College University, Iraq \\ ${ }^{5}$ Middle Technical University, Institute of Technology-Baghdad, Iraq
}

\section{Article Info}

Article history:

Received May 13, 2019

Revised Jun 14, 2019

Accepted Jul 28, 2019

\section{Keywords:}

Light \& heavy load

Multimedia application

OPNET

QoS

WLAN

\begin{abstract}
As the rapid growth of multimedia application over the Internet, it is essential to preserve the Quality of Service (QoS), which is certifying the guaranteed service through the Internet and representing the biggest challenges for the current IP based services. Multimedia traffic usage has been increased in relation to the streaming media such as video conferencing using OPNET, the performance can be simulated based on heavy and light scenarios for video conferencing including web traffic. The overall WLAN load data are obtained for such scenarios, also the performance of simulated overall Delay in the three scenario networks is measured.
\end{abstract}

Copyright () 2020 Institute of Advanced Engineering and Science. All rights reserved.

Corresponding Author:

Adnan H. Ali,

Middle Technical University,

Electrical Engineering Technical College,

Baghdad, Iraq.

Email: dradnanmtu@gmail.com.

\section{INTRODUCTION}

Wireless networking can now be considered as the backbone of the modern telecommunication system. A request for fast transmitting of the high-speed data without appreciable loss had been guided to the technology evolutions such as WLAN or WiMAX. Despite the fact that these technologies have enhancement level that reached for confronting demand from the customers, there is still ample scope for increasing quality of service $(\mathrm{QoS})$ and data rate beyond the present level [1].

The utilizing of wireless networks may be for providing a network connectivity nearly everywhere, such networks equipping big companies with a choice of connecting the wired networks with a modern wireless network unaccompanied by any problems in addition to select any group of applications for users nevertheless of suppliers or sources. The forthcoming WLAN permit users for moving to anyone communication, everyplace at whatever time with a variety services in multimedia. Such applications of wireless communication systems have expanded from simple voice services to integrate data services [2].

The network revolutions, that happening with a technology of broadbands, give a possibility of a wide diversity of applications and facilities. Beyond a market relaxation, an innovative possibility initiates modern applications and requirements. The networks with High-speed permit the conception of original applications, that may cause multiple challenges and big problems. Currently, a commonly using of Wireless networks, but the mobility of users, the management of networks, besides the current protocols 
increase important network issues, that should take into consideration the next-generation in the telecommunication network [3,4].

The wireless networks have multi-services that can transmit data, voices, multimedia, and video traffics [5]. The integration of wireless access and broadband networks in their architecture in order to be convenient for applications of mobile and multimedia with bankrupt traffic [6, 7]. In multimedia networks at high-speed with mobile applications, new challenges have been confused by bankrupt traffic. Actually, the applications of data, multimedia, voice and video network services shared with the challenges in both high-speed and wireless networks need recently developed solutions for synchronization problems in multimedia schemes, dynamic channel function in cellular mobile networks, incoming technologies, QoS and requirements of performance associated with the services and applications proposed [8, 9].

The wireless networking approval is driven by spreading of portable mobile devices, along with an appropriateness of release communications. With growing multimedia content deployment on the Internet along with the deployment of time-sensitive critical applications, there is a strong motivation to develop QoS features to meet the most stringent performance requirements [10]. The term QoS refers to the guarantees on the ability of a network to deliver predictable results and a more deterministic performance, so data can be transferred with a minimum delay, packet loss, jitter and maximum throughput. The QoS does not take into account the user's perception of the quality [11, 12].

The main Internet applications in the wireless network like file transfer or email can be considered elastic applications, that's mean they are not requirements limited by strict performances, and consequently well corresponding with the delivery mode of Internet's datagram which provides network-layer connectionless service [13, 14]. A world of the modern Internet, that is a full capable of multimedia applications, needs guarantees of QoS that users must arise for assuming from the world of telecommunications network. Therefore, for supporting applications of multimedia and other collaborative performances, a support is a needed for QoS features over QoS supplying that delivers resource confirmation along with service distinction $[15,16]$.

The core topical of this work offered in this paper can be focuses in evaluating the WLAN performance, particularly end to end delay, load, media access delay, and the received and sent traffics. Network's multimedia traffic in a video conferencing mode is employed with various scenarios so as to compare between them. The simulation of networks is set up by OPNET 14.5 modeler. Each scenario varies from the others with physical layer technology and transmission rate.

\section{MECHANISM OF RTS/CTS}

The base station in wireless network is well prepared for transmitting and made sending a short frame of Request To Send (RTS) before transmission of each data frame. An RTS frame impact is less potential than impact of the real data frame owing to the size differences. When the base station at the receiver is ready for receiving, the RTS frame is acknowledged with a send frame of Clear To Send (CTS) for sender, hence all traffic are blocked from another station [17]. Furthermore, if the source is received CTS frame, then a data frame is sent in case channel is reserved for a full transmission length. Finally, the ACK frame is sent by receiver to the sender based on frame receiving [18]. Thus, a significant efficiency evaluation of optional mechanism of RTS/CTS handshake on the performance for analyzing the IEEE 802.11 based WLANs [19].

\section{OPNET IMPLEMENTATION \& SIMULATION}

\subsection{OPNET Modulator}

OPNET Company (Optimized Network Engineering Tool) was initiated in MIT (Massachusetts Institute of Technology), and its establishment in 1986. A year later, 1987, first simulation software of commercial network performances was released by OPNET company which could providing an optimization tool of important network performances that made a revolutionized network simulation [20]. Creation the management of analytical network performances is powerful with simulation and thus it becomes possible. The development of other products at OPNET besides Modeler is done, it also comprises a Kit of OPNET Development, WDM Guru etc [21, 22].

The model of simulation is considered a progressively popular approach for studying the performances and functionalities of the suggested models in various scenarios. Simulation is a testing procedure of a designed prototypical on a platform that duplicates the real environment and offers the opportunity for studying, creating, and modifying the performance of design proposing with the purpose of strengthens and weakness the expectation before the model implementation in a real environment [23, 24]. 
A flow chart of simulation of any system started with the steps that is taken in the simulation process. For each scenario, the network traffic, configurations are modified and the simulation is run [25]. A flowchart is used to illustrate the key steps taken to assess the performances of a WLAN is seen in Figure 1.

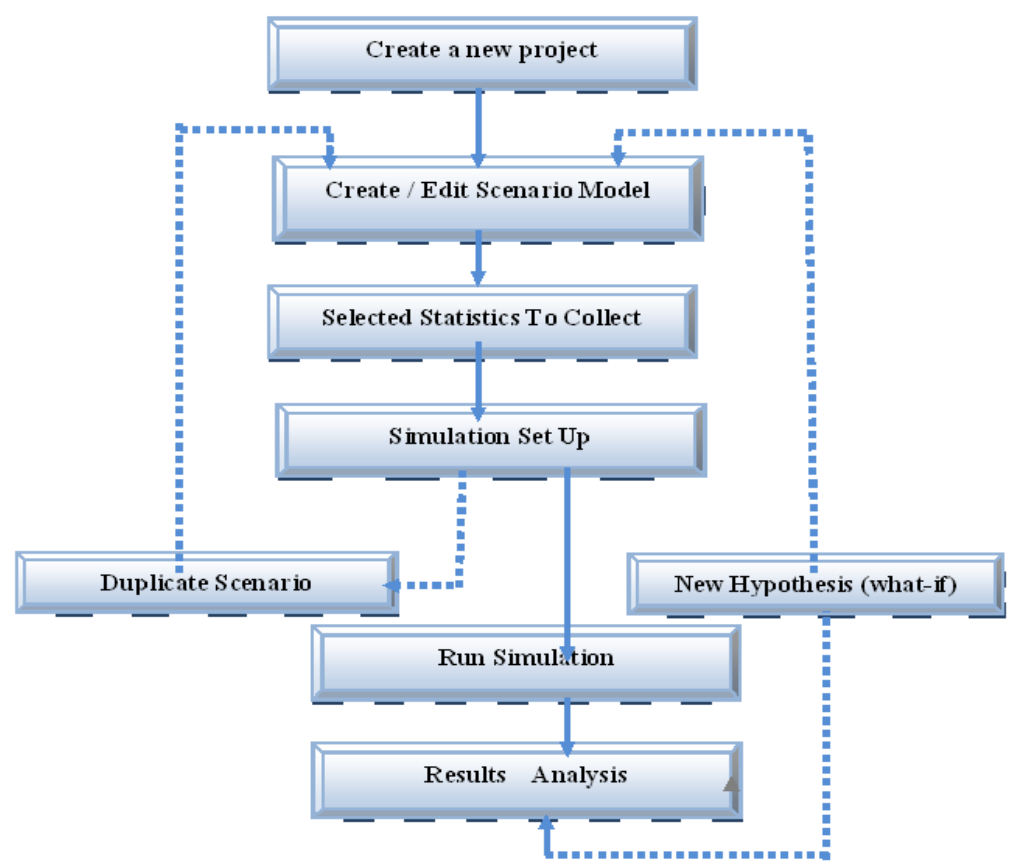

Figure 1. Flow chart to evaluate the performance of WLAN

\subsection{Baseline Scenario}

The Baseline 802.11g model scenario is formed utilizing a standard model's variation of the OPNET 14.5 WLAN arrangement scenario. Within such scenario, a single infrastructure $802.11 \mathrm{~g}$ WLAN behavior can be inspected in the organized WLAN framework for better emulating the actual network conformation as shown in Figure 2. An Internet Protocol IP cloud van be using to signify the Internet backbone is connected with a serial link of Point-to-Point T1 (1.544Mbps) [19]. The three subnets are positioned at each side of IP cloud through an IP gateway connecting by Point-to-Point Protocol T1 link and two servers are connecting via a central switch utilizing a link of 100BaseT, as seen in Figure 2, and wireless as seen in Figure 3 respectively.

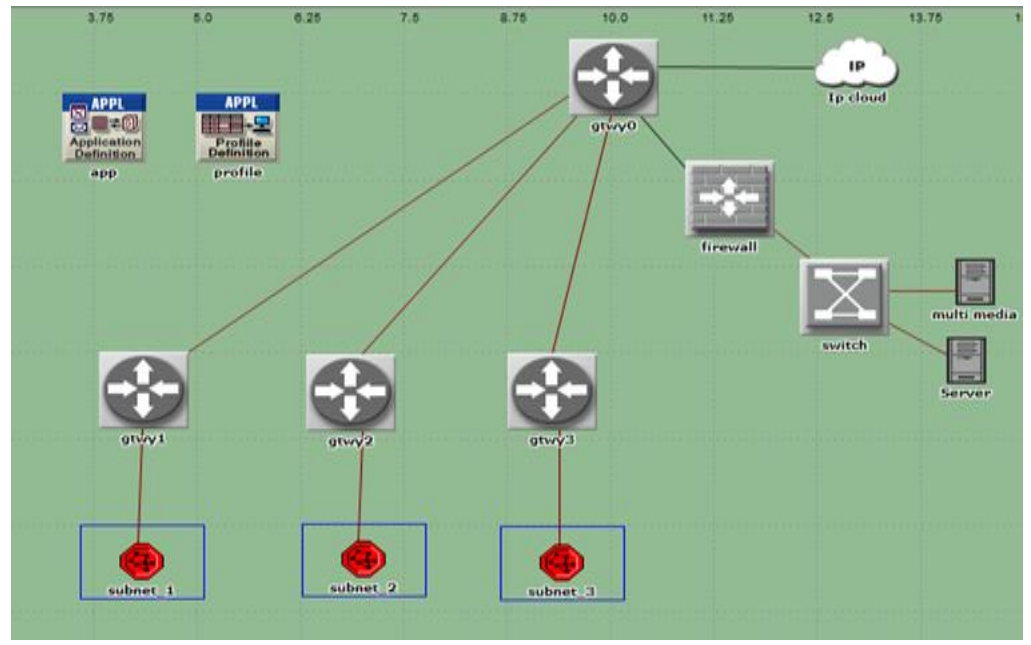

Figure 2. Simulated wire server frameworks 


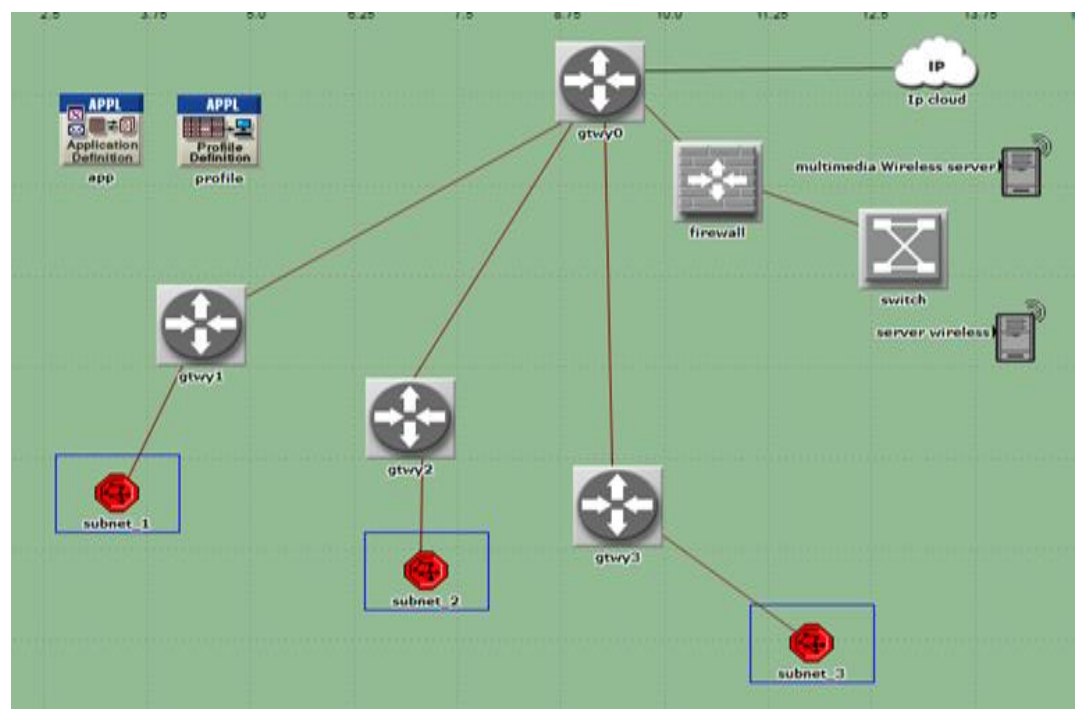

Figure 3. Simulated wireless server frameworks

The location of subnet one is at the right side of the Internet Protocol (IP) cloud and the servers of the traffic network are connected by 100BaseT Ethernet, the servers is connecting to the firewall by 100BaseT Ethernet link and can be using in place of sources and destinations at all applications: video conferencing, Hypertext Transfer Protocol (HTTP), File Transfer protocol (FTP), voice applications, Electronic Mail (E-mail) and Database simulation on the completed network characterizing the traffic which exchanges with the 802.11g WLAN mobile nodes through simulations, as shown in Figure 4.

The subnet two denotes the office of remoting branch that comprising five workstations in the office_LAN connecting by a link of 100BaseT. This LAN office is connecting by a central switch with a link 100Base Ethernet wiring to compete with a real-time office situation which uses Fast Ethernet LAN standards. The IP gateway connects the LAN to an IP cloud, this gateway is connected to the LAN office by 100BaseT Ethernet link whereas the serial link P2P T1 is connect the IP cloud and the IP gateway, as seen in Figure 5.

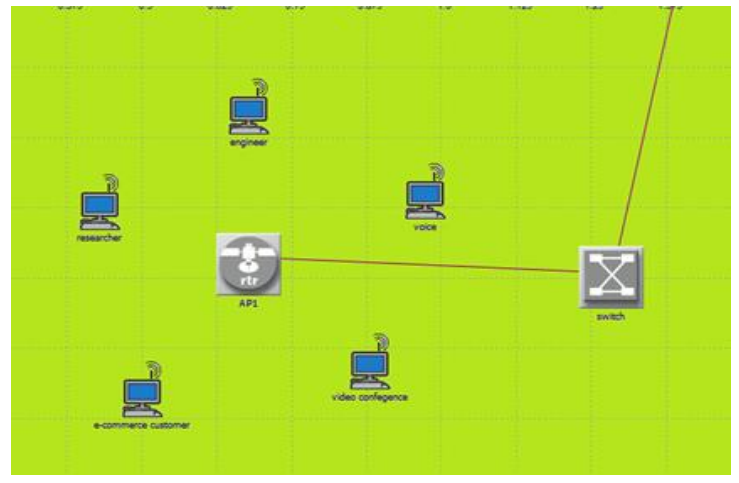

Figure 4. Subnet $1802.11 \mathrm{~g}$ WLAN

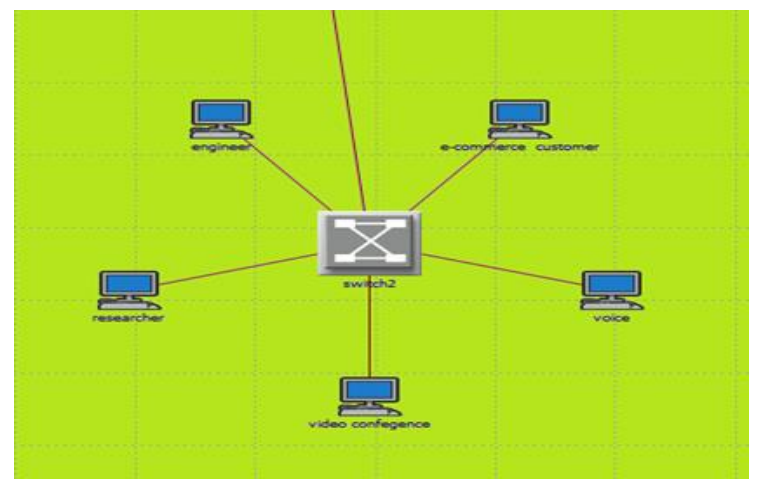

Figure 5. Subnet 2 802.11g WLAN

Finally, the subnet three located on the other side of the Internet Protocol cloud, a connection of WLAN is done within access point AP to LAN office by a central switch utilizing Ethernet wiring to emulate an environment of real-time office within Fast Ethernet LAN standards that has extension of a WLAN to a zone of wiring difficulty or demanding aesthetics like a media (voice or video) room or conference, as shown in Figure 6. The office_LAN are divided equally amongst the profiles, with each profile having one workstation. Here the main objective is to analyses the performances of operator perceived usefulness belong to WLAN users, An WLAN sub network with five mobile nodes are assigned to different profiles as shown in Table 1. 


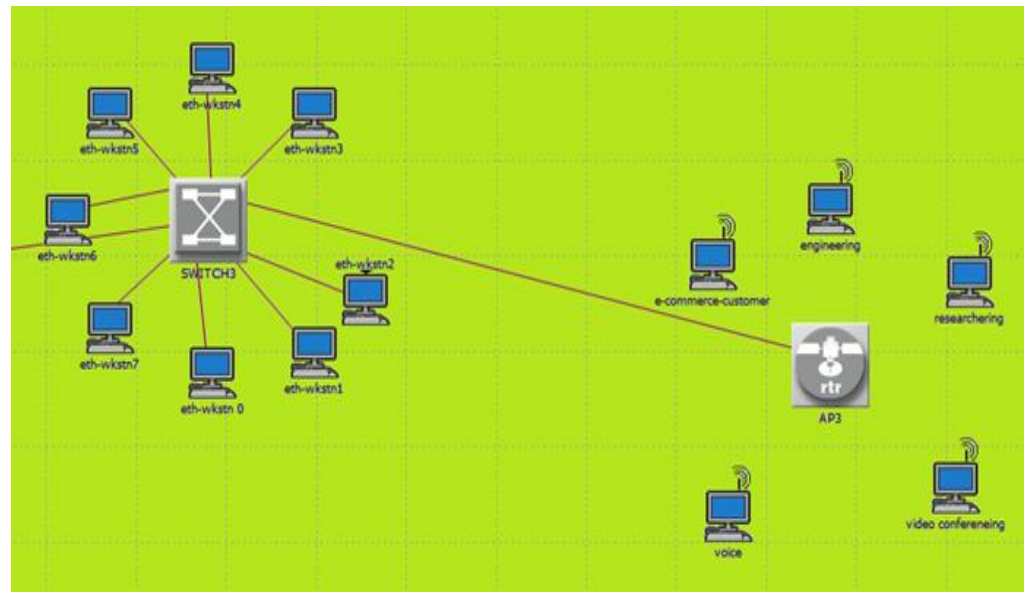

Figure 6. Subnet 3 Offices WLAN

Table 1. Assigned Profiles of Mobile Nodes in the WLAN Sub Network

\begin{tabular}{cc}
\hline MOBILE NODE & USER PROFILE \\
\hline Station_1 & Engineer \\
Station_2 & Researcher \\
Station_3 & E-commerce Customer \\
Station_4 & Sales Person \\
\hline
\end{tabular}

\subsection{Multimedia Load Scenarios}

The multimedia traffic can be represented as a video conferencing form in the network. This form of conferencing involves images, data, and voice, and definitive multimedia traffic representation. While the video server is presented for assisting the application of video conferencing towards the details of network, Figure 7 shows the configuration for multimedia employer profile applications at the scenarios of multimedia load.

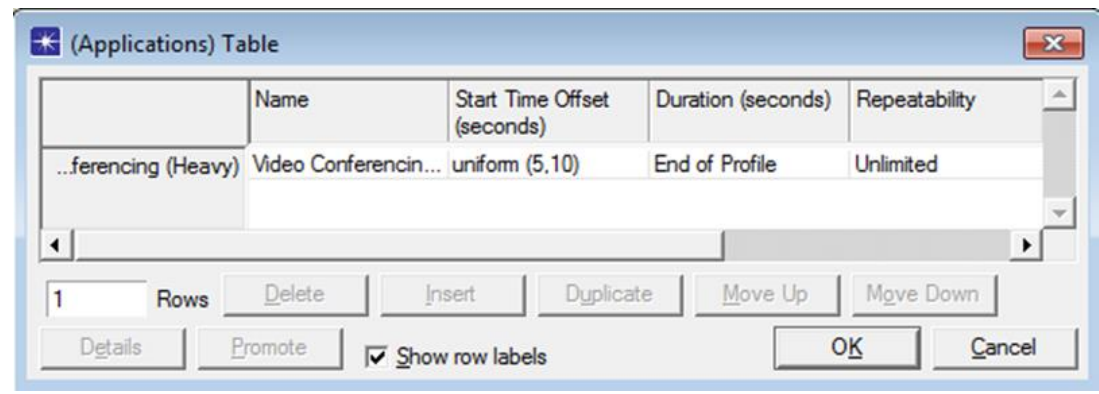

Figure 7. Application Configurations for Multimedia User Profile

\section{SIMULATION RESULTS AND DISCUSSIONS}

\subsection{Load}

A load is the first parameter which effects the total performance of the wireless efficiency. The assessment of the load is concerned with the reception of the transmitted data in spite of don't consider the network overhead opposed to the load. A total WLAN load with respect to the time function as the advanced simulation can be considered as a greater extent significant results, Data have overall WLAN load with an approximate average value of scenario heavy is (430.7407) Kbps on the 5 minute mark and the average value of scenario light WLAN is (3622787) Kbps on the 5 minute mark and Figure 8 shows the results belong to the first scenario. A simulation scenario (heavy) was built and run in order to obtain the desired results for individual load as shown in Figure 9. 


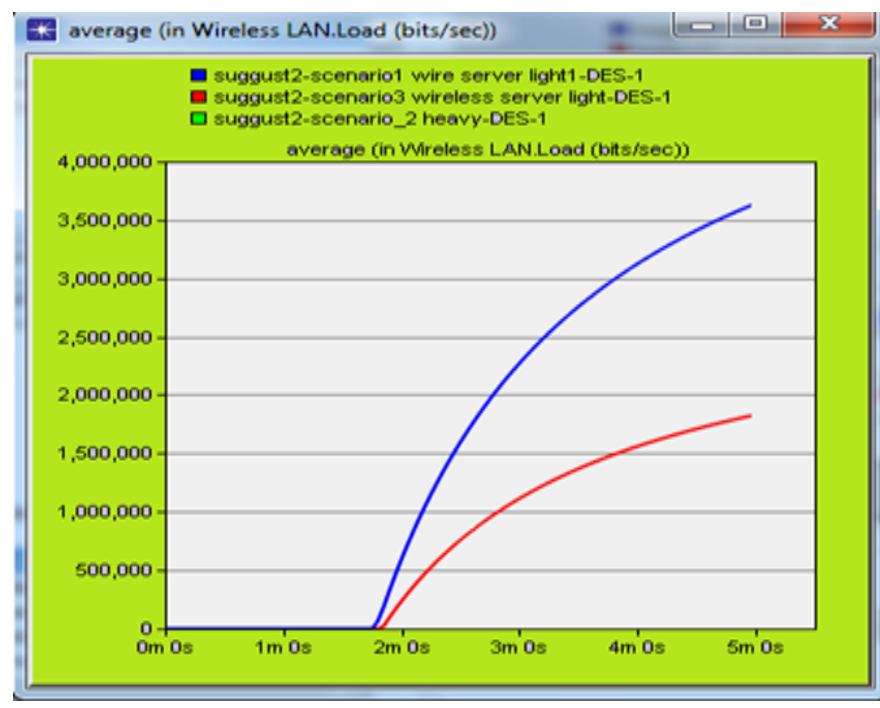

Figure 8. Total Loads on the Simulated Wireless LAN

suggust2-scenario_2 heavy-DES-1: average (in Wireless... $\left[\begin{array}{c|}\text { a } \\ \hline\end{array}\right.$

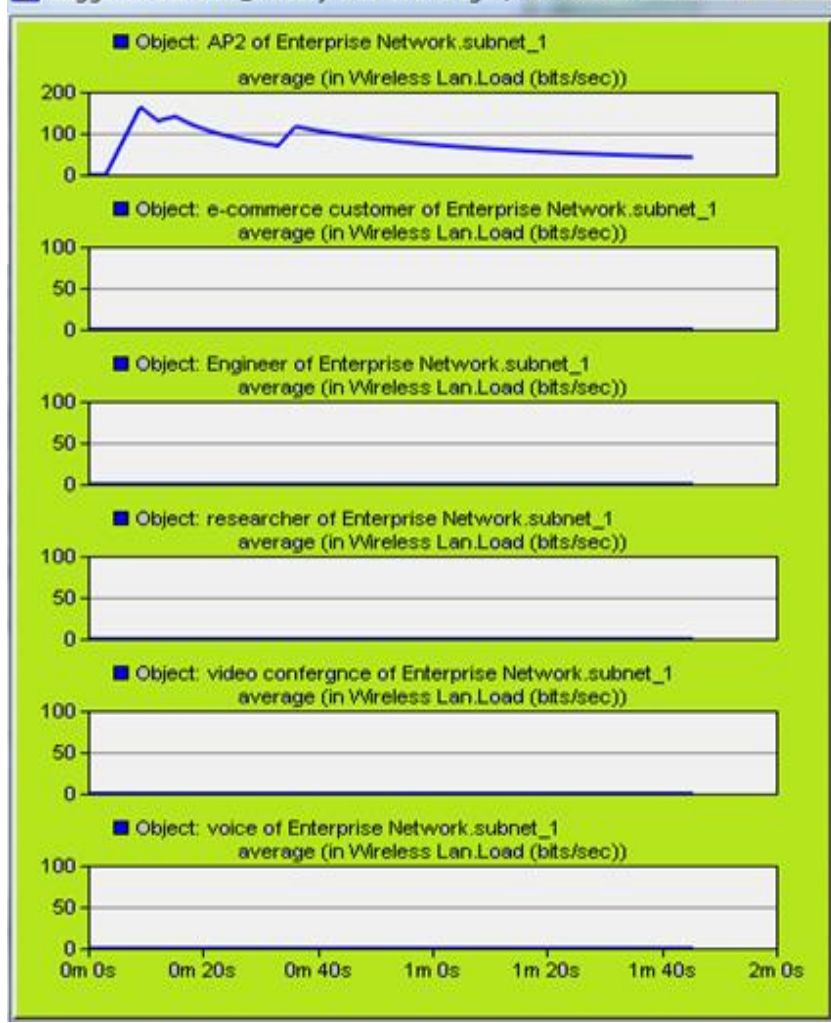

suggust2-scenario_2 heavy-DES-1: average (in Wireless... 0 미밀

- Object. AP1 of Enterprise Network.subnet_3

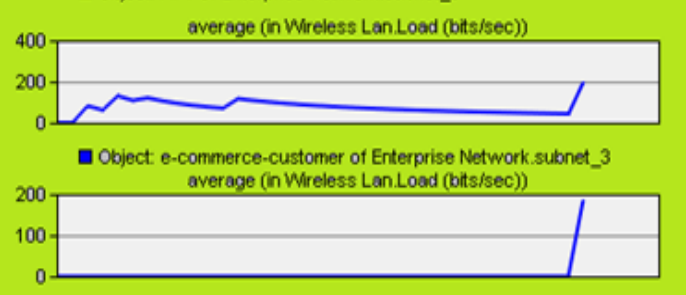

- Object: engineering of Enterprise Network.subnet_3

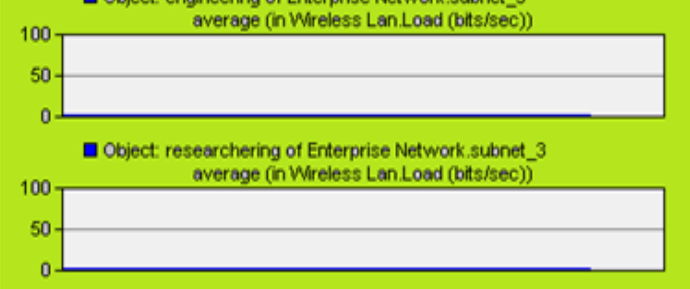

- Object video confereneing of Enterprise Network.subnet_3

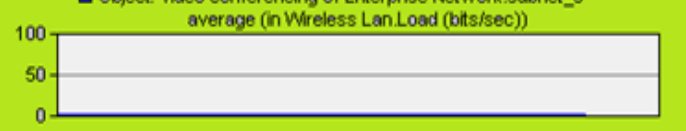

- Object: voice of Enterprise Network. subnet 3 average (in Wireless Lan Load (bits/sec))

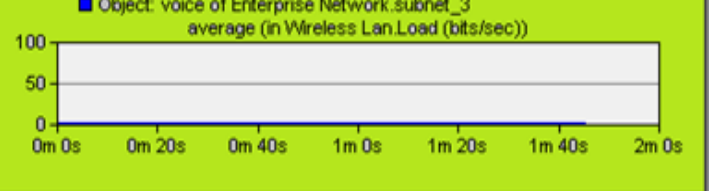

Figure 9. Individual load values

A simulation scenario (Light) was built and run in order to obtain the results for Individual Load Values for a subnet, as shown in Figure 10. In order to obtain the desired results of the load and the access point are illustrated approximate average peak values stations to achieve the objective, A simulation scenario (heavy) with a simulation scenario (Light) can be represented in Table 2. 

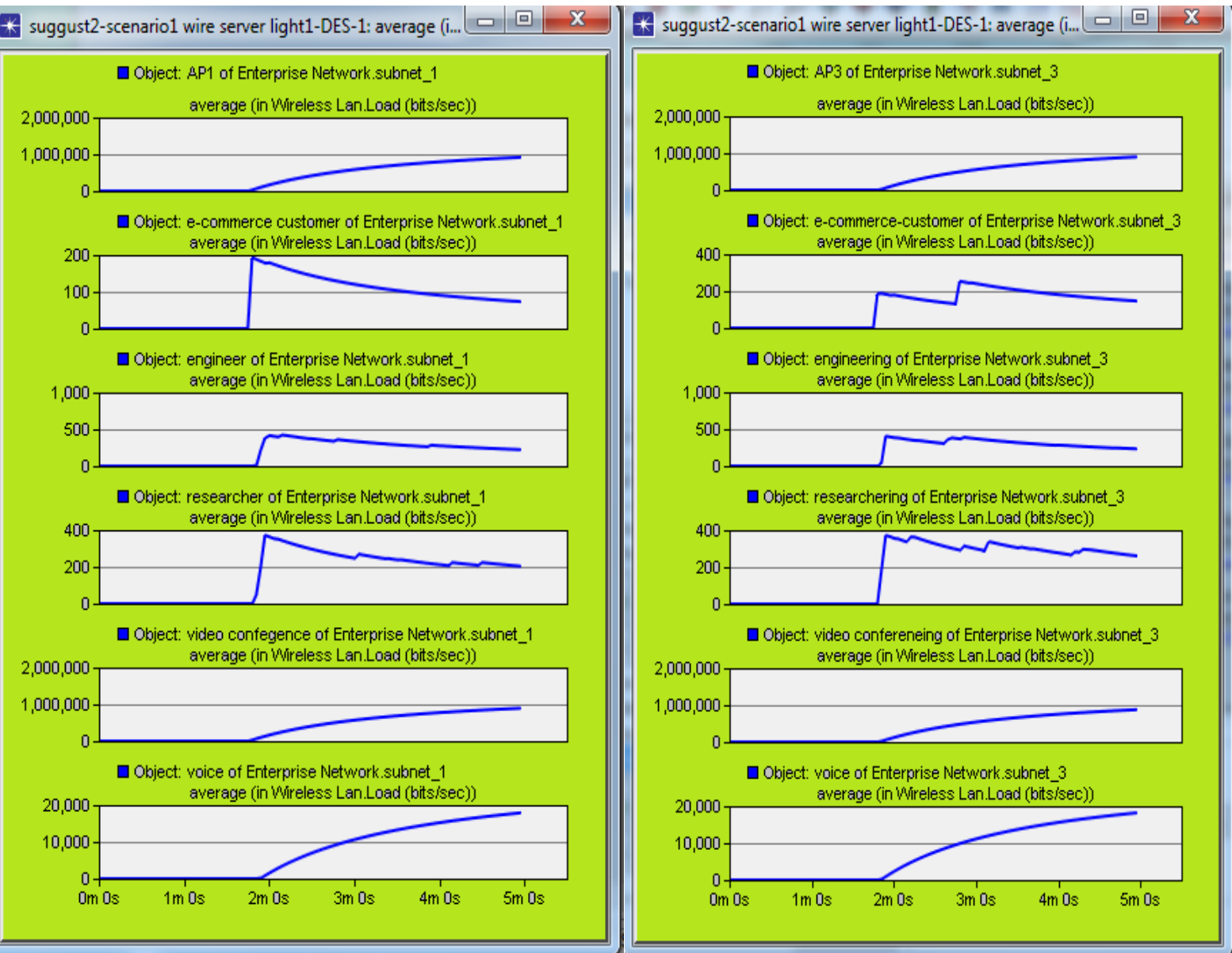

Figure 10. Individual load values for scenario (light) subnet one, subnet three

Table 2 The Individual load values scenario 1(light) and scenario 2(heavy)

\begin{tabular}{lcccc}
\hline \multicolumn{1}{c}{ Type Of Node } & $\begin{array}{c}\text { WLAN Load } \\
\text { (Heavy) (Kbps) } \\
\text { subnet one }\end{array}$ & $\begin{array}{c}\text { WLAN Load (Heavy) } \\
\text { (Kbps) subnet three }\end{array}$ & $\begin{array}{c}\text { WLAN Load } \\
\text { (Light) (Kbps) } \\
\text { subnet one }\end{array}$ & $\begin{array}{c}\text { WLAN Load (Light) (Kbps) } \\
\text { subnet three }\end{array}$ \\
\hline Access point & (AP2) 42.07 & (AP1) 200.2 & (AP1) 916124 & (AP3) 899128 \\
$\begin{array}{l}\text { E-commerce } \\
\text { costumer }\end{array}$ & 0 & 188.4 & 73 & 146 \\
$\begin{array}{l}\text { Engineer } \\
\text { Researcher }\end{array}$ & 0 & 0 & 223 & 232 \\
$\begin{array}{l}\text { Video } \\
\text { conferencing }\end{array}$ & 0 & 0 & 202 & 260 \\
$\begin{array}{l}\text { Voice } \\
\text { Sub Value }\end{array}$ & 0 & 0 & 893783 & 87652 \\
Sum total & 42.07 & 0 & 17878 & 18201 \\
\hline
\end{tabular}

\subsection{Delay}

Delay can consider fundamental metric for describing any network's QoS, specifically a real time. In application of multimedia, delay may perform a central parameter for choosing the MAC layer effective operation, its operation time, and the mechanism of Required To Send/ Clear To Send (RTS/CTS). Delay has two main kinds, they are a medium access delay and overall packet transmission delay statistics, Figure 11 shows a results of average overall WLAN delay peaks at $0.0125 \mathrm{~s}$, these values are distinctive of an efficient WLAN under normal traffic loads, while the average (in the Video conferencing packet End-to-End Delay (sec)) delay peaks at (0.055s) as seen in Figure 12.

It can be seen that an WLAN Delay which characterizes the end to end delay of all the packets received by the wireless LAN MACs by all nodes of WLAN network then higher layer forwarded. This delay comprises medium access delay at the source MAC, reception of all the fragments individually. An indication that WLAN Delay can be considered very high throughout those resends. The average (in the 
Voice packet End-to-End Delay (sec)) delay peaks at (0.093s) as shown in Figure 13. A simulation different WLAN Delay of applications are used and simulation scenario 1 (Light) Wire Server $(0.0103(\mathrm{sec}))$ vs Wireless Server $(0.0125(\mathrm{sec}))$ as shown in Figure 14. The delay and the access point are illustrated approximate average peak values as shown in Table 3.

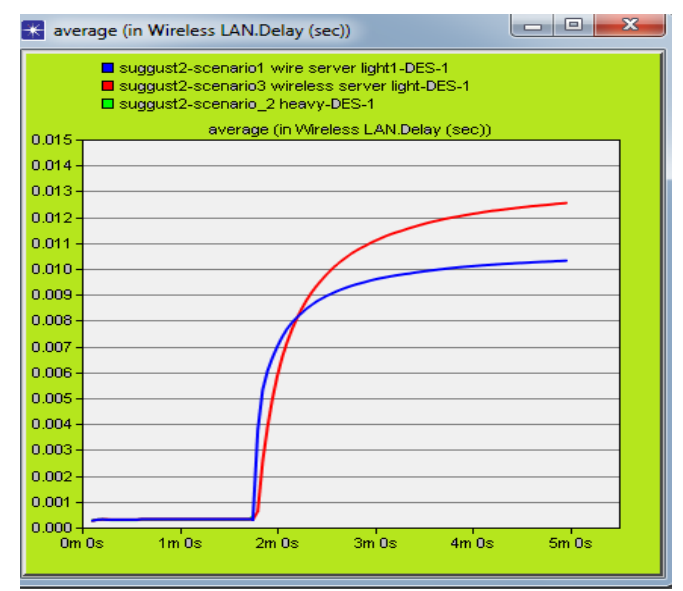

Figure 11. Simulated overall delay

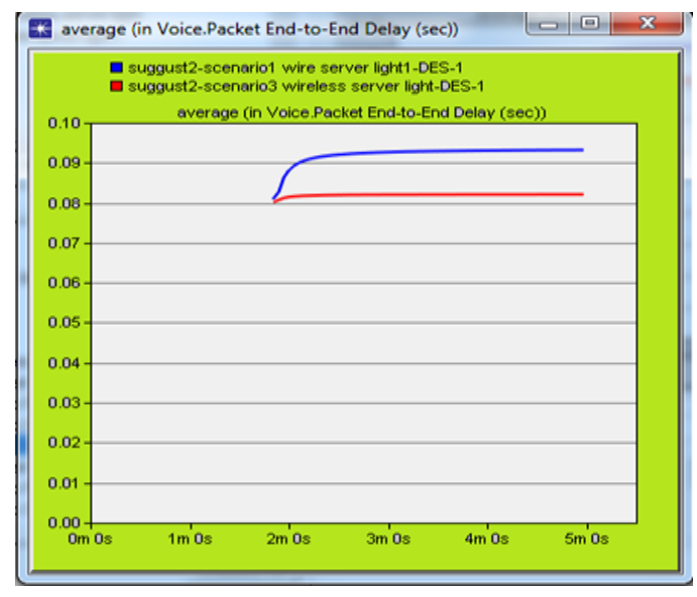

Figure 13. Simulated voice wire server delay vs wireless server delay

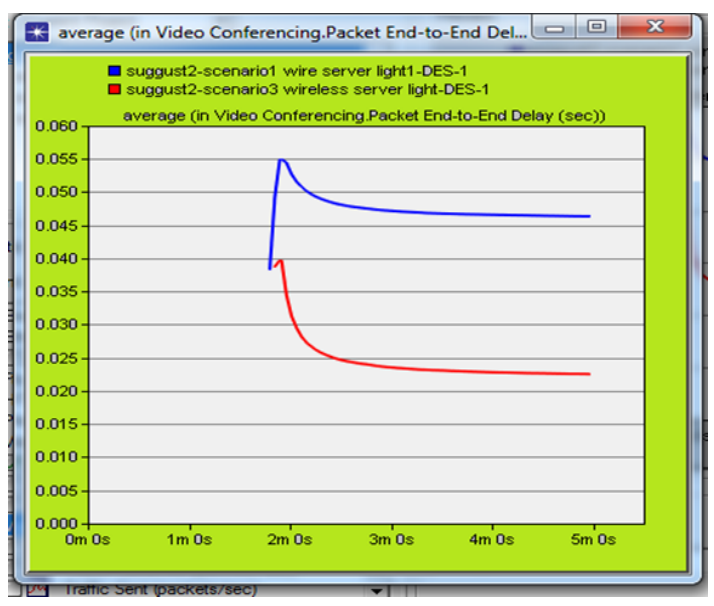

Figure 12. Simulated video conferencing wire

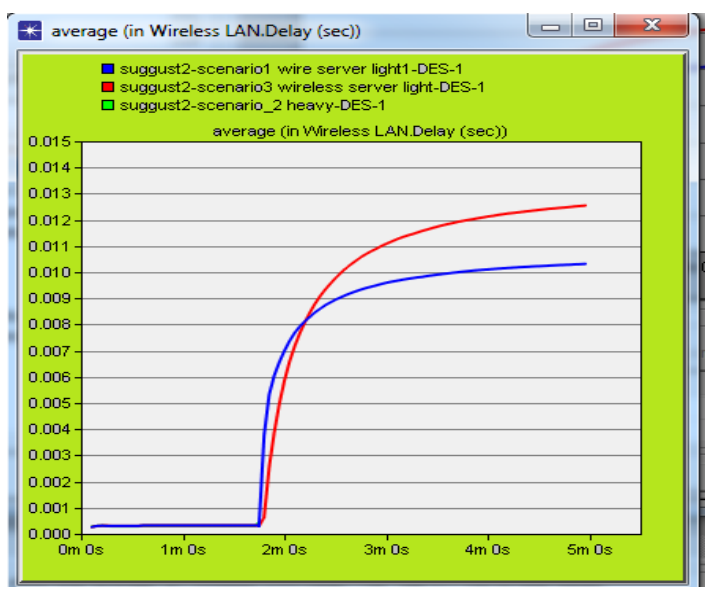

Figure 14. Simulated WLAN delay (sec) wireless servers vs wire server

Table 3. Wireless LAN delay (sec) Server of applications in WLAN

\begin{tabular}{ccc}
\hline Application & $\begin{array}{c}\text { Wireless server Delay } \\
(\mathrm{sec})\end{array}$ & $\begin{array}{c}\text { Wire server Delay } \\
(\mathrm{sec})\end{array}$ \\
\hline $\begin{array}{c}\text { Video } \\
\text { conferencing }\end{array}$ & 0.039 & 0.055 \\
Voice & 0.082 & 0.093 \\
\hline
\end{tabular}

\section{CONCLUSION}

The performances of Quality of Service QoS can apply in IP networks for employment of available network resources at greatest efficient method for minimizing delays in network traffic that have multiple forms of multimedia services which contains voice, video and database. Two network scenarios have been created, namely 1 heavy network, Scenario 2 (Medium Load) network and Scenario 3 (High Load) network. The Comparison was carried out between them. Performance assessments directed were both Loads and Delays Performances, in these Performance tests, we observed the results in two cases: heavy and light scenarios. 


\section{REFERENCES}

[1] Sanam Narejo, Farzana Kulsoom, M. Moazzam Jawaid," Performance Evaluation of Interactive Video Streaming over WiMAX Network", International Journal of Electrical and Computer Engineering (IJECE) Vol. 7, No. 1, February 2017, pp. 344 352.

[2] Shuang Song and Biju Issac, "Analysis of Wifi And Wimax And Wireless Network Coexistence" International Journal of Computer Networks \& Communications (IJCNC) Vol.6, No.6, November 2014.

[3] Mohammed, H.A., Ali, A.H., Mohammed, H.J. The Affects of Different Queuing Algorithms within the Router on QoS VoIP Application using OPNET. International Journal of Computer Networks \& Communications, 2013. 5(1): p. 117-124.

[4] M.S. Borella, Source models of network game traffic, Computer Communications 23 (2000) 403-410.

[5] Fabien Houe'to, Samuel Pierre" Quality of service and performance issues in multiservice networks subject to voice and video traffics", Computer Communications 28 (2005) 393-404

[6] A. Malik, J. Qadir, B. Ahmad, K. A. Yau, and U. Ullah, "QoS in IEEE 802.11-based Wireless Networks: A Contemporary Survey", preprint available at http://arxiv.org/abs/1411.2852,

[7] Hussein A. Mohammed, Adnan H. Ali, "Effect of some Security Mechanisms on the Qos VoIP Application using OPNET", International Journal of Current Engineering and Technology, Vol.3, No.5, December, 2013.

[8] Luo and Shyu, "Quality of service provision in mobile multimedia - a survey", Computing and Information Sciences, 1: 5, 2011 .

[9] Malik, A., Qadir, J., Ahmad, B., Alvin Yau, K.-L., \& Ullah, U. (2015). QoS in IEEE 802.11-based wireless networks: A contemporary review. Journal of Network and Computer Applications, 55, 24-46.

[10] Ali A. Abdulrazzaq, Adnan H. Ali, "Efficiency Performances of Two MPPT Algorithms for PV System with Different Solar Panels Irradiances", International Journal of Power Electronics and Drive System (IJPEDS) Vol. 9, No. 4, December 2018, pp. 1755 1764.

[11] K. Xu, M. Gerla and S. Bae, "Effectiveness of RTS/CTS Handshake in IEEE 802.11 Based Ad Hoc Networks", Ad Hoc Networks Journal, vol. 1, no.1, pp. 107-123, July 2003.

[12] Abdulrazzaq, A.A.; Abid, A.J.; Ali, A.H. QoS Performances Evaluation for Mobile WIMAX Networks based on OPNET. Int. J. Appl. Eng. Res. 2018, 13, 6545-6550.

[13] Anindita Kundu1, Iti Saha Misra2, Salil K. Sanyal3, Suman Bhunia, "Voip Performance Over Broadband Wireless Networks Under Static and Mobile Enviroments", International Journal of Wireless \& Mobile Networks (IJWMN) Vol.2, No.4, November 2010.

[14] Adnan H. Ali, Farhood, A.D. Design and Performance Analysis of the WDM Schemes for Radio over Fiber System with Different Fiber Propagation Losses. Fibers 2019, 7, 19.

[15] Adnan H. Ali,Ali N. Abbas, M. H. Hassan," Performance Evaluation of IEEE802.11g WLANsUsing OPNET Modeler", (AJER) Volume-02, Issue-12, pp-09-15.

[16] O. Aboul-Magd, Wireless Local Area Networks Quality of Service: An Engineering Perspective. IEEE, 2009.

[17] Adnan H. Ali, Hayder J. Alhamdane, Begared S. Hassen, "Design analysis and performance evaluation of the WDM integration with CO-OFDM system for radio over fiber system", Indonesian Journal of Electrical Engineering and Computer Science IJEECS, Vol. 15, No. 2, August 2019, pp. 870 878.

[18] K. Xu, M. Gerla and S. Bae, "How Effective is the IEEE 802.11 RTS/CTS Handshake in Ad Hoc Networks?", in Proc. of IEEE Globecom '02, vol. 1, pp. 72-76, Taipei, 2002.

[19] Adnan H. Ali, 2015 Performance Evaluation of Wi-Fi Physical Layer Based QoS Systems on Fiber Using OPNET Modeler International Journal of Soft Computing and Engineering (IJSCE) 5 Issue-3.

[20] M. M. Kareem, M. Ismail, M. A. Altahrawi, N. Arsad, M. F. Mansor, and A. H. Ali, 'Grid Based Clustering Technique in Wireless Sensor Network using Hierarchical Routing Protocol', in 2018 IEEE 4th International Symposium on Telecommunication Technologies (ISTT), 2018, pp. 1-5.

[21] M. I. Youssef, A. E. Emam, M. Abd Elghany, "Image multiplexing using residue number system coding over MIMO-OFDM communication system", International Journal of Electrical and Computer Engineering (IJECE) Vol. 9, No. 6, December 2019, pp. 4815 4825.

[22] Ali, A.H., Abdul-Wahid, S.N.: Analysis of self-homodyne and delayed self-heterodyne detections for tunable laser source linewidth measurements. IOSR J. Eng. 2(10), 1-6 (2012).

[23] Ahmed J. Abid, Fawzi M. Al-Naima, and Adnan H. Ali, "Comprehensive Modeling of PV Array based on Proteus Software", IJAER, Volume 13, Number 6 (2018) pp. 4440-4447.

[24] Haeeder M. Noman, Ali A. Abdulrazzaq, Marwah M. Kareem, and Adnan H. Ali, "Improvement Investigation of the TCP Algorithms with Avoiding Network Congestion Based on OPNET", 2nd ICSET 2019, IOP Conf. Series: Materials Science and Engineering (2019).

[25] A.D. Farhood, Naji, M.K., Rhaif, S.H., Ali A.H., "Design and analysis of dual band integrated hexagonal shaped microstrip UWB antenna" , Indonesian Journal of Electrical Engineering and Computer Science IJEECS, 2019, Volume 15, Issue 1, Pages 294-299.

[26] Ali A. Abdulrazzaq, Adnan H. Ali, "Performance Investigation of Grid Connected Photovoltaic System Modelling Based on MATLAB Simulation", IJECE 8 (6), 2018. 